TecnoHumanismo. Revista Científica

Setiembre - Noviembre 2021

Volumen 1 / No.3

ISSN: $2710-2394$

pp.1-11

HUMANISMO

https://doi.org/10.53673/th.v1i12.79

https://tecnohumanismo.online

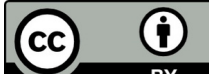

\title{
Evaluación del nivel en la provisión de servicios públicos en la ciudad de Huánuco - 2018
}

Evaluation of the level in the provision of public services in the city of Huánuco - 2018

Avaliação do nível de prestação de serviços públicos na cidade de Huánuco - 2018

\section{ARTÍCULO GENERAL}

\author{
Manuel Marín Mozombite \\ https://orcid.org/0000-0003-4537-7787 \\ mmarin@unheval.edu.pe
}

Universidad Nacional Hermilio Valdizán, Huánuco - Perú

Recibido 07 de Abril 2021 | Arbitrado y aceptado 19 de Junio 2021 | Publicado en 03 Setiembre 2021

\section{RESUMEN}

Esta investigación 2018 es una extensión de un proyecto iniciado el año 2017 , más ambicioso, al haber coberturado once instituciones de la ciudad de Huánuco en relación a la provisión de servicios públicos institucionales, además por haber extendido esa cobertura hasta servicios domiciliarios y servicios extra domiciliarios, que fueron evaluados en hogares y en el mismo punto de consumo, como ocurrió en el caso del transporte urbano. La investigación 2017 evaluó el nivel de servicio en la Universidad Nacional Hermilio Valdizán, contextualizado en la denominación de ese año, "Año del Buen Servicio al Ciudadano". El problema general planteado ha sido ¿Cuál es la valoración de los principales servicios públicos que se brinda en la ciudad de Huánuco?; esta investigación está en relación al nivel de servicio y tres han sido las categorías de servicio investigados: los que brindan las instituciones públicas, denominados servicios eventuales; servicios domiciliarios como el agua potable y la energía eléctrica; $y$, servicios extra domiciliarios como el serenazgo, el recojo de basura y el transporte urbano. Los resultados se presentan según los objetivos específicos, el nivel de servicio de instituciones públicas, el nivel de servicio de los servicios domiciliarios y extra domiciliarios; como conclusiones relevantes se anotan que son dos instituciones públicas las que destacan por su buena performance en cuanto a los servicios que prestan, con niveles de servicio entre $77 \%$ y $80 \%$, así como las dos de peor performance reportan niveles de entre $51 \%$ y $53 \%$, y que de entre cinco servicios domiciliarios y extra domiciliarios, cuatro de ellos apenan alcanzan entre $38 \%$ y $51 \%$ de nivel de servicio, que según la escala de valoración de resultados son percibidos como regular y en estado crítico. Concluye el informe presentando las brechas de servicio en relación a un estado deseado de $100 \%$ y en base a ellas, las recomendaciones pertinentes.

Palabras clave: Servicio, Nivel de Servicio, Brecha del Servicio

\section{ABSTRACT}

This 2018 research is an extension of a project started in 2017, more ambitious, having covered eleven institutions in the city of Huánuco in relation to the provision of institutional public services, in addition to having extended that coverage to home services and extra-home services which were evaluated in homes and at the same point of consumption, as in the case of urban transport. The 2017 research evaluated the level of service at the Hermilio Valdizán National University, contextualized in the name of that year, "Year of Good Citizen Service". The general problem raised has been: What is the assessment of the main public services provided in the city of Huánuco ?; This research is in relation to the level of service and there have been three categories of service investigated: those provided by public institutions, called occasional services; home services such as drinking water and electricity; and, extra-domiciliary services such as serenazgo, garbage collection and urban transport. The results are presented according to the specific objectives, the level of service of public institutions, the level of service of home and extra-home services; As relevant conclusions, it is noted that two public institutions stand out for their good performance in terms of the services they provide, with service levels between $77 \%$ and $80 \%$, as well as the two with the worst performance report levels between $51 \%$ and $53 \%$, and that out of five home and extra home services, four of them distressed reach between $38 \%$ and $51 \%$ of service level, which according to the results assessment scale are perceived as regular and in critical condition. The report concludes by presenting the service gaps in relation to a desired state of $100 \%$ and based on them, the pertinent recommendations.

Keywords: Service, Service Level, Service Gap

\section{RESUMO}

Esta pesquisa de 2018 é uma extensão de um projeto iniciado em 2017, mais ambicioso, tendo abrangido onze instituições da cidade de Huánuco no que diz respeito à prestação de serviços públicos institucionais, além de ter alargado essa cobertura aos serviços ao domicílio e aos serviços extra domiciliários., que foram avaliados nas residências e no mesmo ponto de consumo, como no caso do transporte urbano. A pesquisa de 2017 avaliou o nível de serviço da Universidade Nacional Hermilio Valdizán, contextualizado no nome daquele ano, "Ano do Bom Atendimento ao Cidadão". O problema geral levantado foi: Qual a avaliação dos principais serviços públicos prestados na cidade de Huánuco ?; Esta pesquisa é em relação ao nível de serviço e foram investigadas três categorias de serviços: os prestados por instituições públicas, denominados serviços ocasionais; serviços domésticos, como água potável e eletricidade; e, serviços extradomiciliares como serenazgo, coleta de lixo e transporte urbano. Os resultados são apresentados de acordo com os objetivos específicos, o nível de serviço das instituições públicas, o nível de serviço dos serviços domiciliários e extra domiciliares; Como conclusões relevantes, nota-se que duas instituições públicas se destacam pelo bom desempenho ao nível dos serviços que prestam, com níveis de serviço entre $77 \%$ e $80 \%$, bem como as duas com piores níveis de relatório de desempenho entre $51 \%$ e $53 \%$, sendo que dos cinco serviços domiciliares e extra domiciliares, quatro deles atingem entre $38 \%$ e $51 \%$ do nível de serviço, que pela escala de avaliação de resultados são percebidos como regulares e em estado crítico. $\mathrm{O}$ relatório conclui apresentando as lacunas do serviço em relação a um estado desejado de $100 \%$ e com base nelas, as recomendações pertinentes. Palavras-chave: serviço, nível de serviço, lacuna de serviço 


\section{Introducción}

Calidad, servicio, nivel de servicio o calidad de servicio son términos recurrentes en nuestra cotidianidad referidos muchas veces en forma casi indistinta, sin embargo, sin ser en extremo diferentes cada una de ellas refiere algo diferente y también comparten, por lo menos, la intencionalidad inherente de lo que se quiere transmitir. En el orden nombrado y con sinergia de tantas definiciones, la primera es una aspiración de todo lo que hacemos o recibimos, el segundo es una variante de lo que se llama producto y todos o brindamos o recibimos, el nivel de servicio o calidad de servicio son apreciaciones o valoraciones en escala adecuada.

Todo, hoy en día se valora como servicio, los bienes en la última etapa de transacción se juzga como servicio de venta, los procesos, se dice, se valoran en términos de servicios destacando la interacción de ofertantes y demandantes, y en este marco se destaca que desde hace por lo menos dos décadas atrás los intentos por medir la calidad, en un comienzo solo atribuidos a bienes tangibles, se tuvieron que extender hasta los servicios; si bien al comienzo era muy extensa el ámbito de su valoración o medición en lo concerniente a servicios, a la fecha se han extendido procedimientos más sistematizados donde destaca la propuesta o metodología SERVQUAL, base de la implementación de esta investigación. Con base en esta metodología, esta investigación ha extendido su cobertura hasta servicios domiciliarios y extra domiciliarios, adecuando los constructos para su valoración

La participación de usuarios de los diferentes servicios ha sido una fortaleza a la hora del trabajo de campo, en todos los casos, se encontró predisposición para aplicar las encuestas, quizás la investigación fue su oportunidad para dar a conocer su opinión; también se destaca la participación de los alumnos colaboradores, todos del curso Planeamiento de Control de Operaciones I, ciclo 2018 I, tal como se reconoce en los créditos de la carátula.

\section{Marco metodológico.}

Tipo de estudio: Exploratorio y transversal

Nivel de estudio: Descriptivo y explicativo. 
Población: La población referenciada para la investigación han sido personas, usuarios efectivos $^{1}$ de algún tipo de servicio, caracterizados según:

a. Instituciones públicas

\begin{tabular}{|l|l|}
\hline Seda Huánuco & Dirección Regional de Educación \\
\hline Essalud & Electrocentro \\
\hline Dirección Regional de Trabajo & Municipalidad Provincial Huánuco \\
\hline RENIEC & Gobernación Regional Huánuco \\
\hline Municipalidad Distrital Pillco Marca & Municipalidad Distrital Amarilis \\
\hline Dirección Regional de Transportes & \\
\hline
\end{tabular}

b. Hogares urbanos

\begin{tabular}{|l|l|l|}
\hline Distrito de Huánuco & Distrito de Amarilis & Distrito de Pillco Marca \\
\hline
\end{tabular}

c. Arribo y embarque de transporte urbano

\begin{tabular}{|l|l|}
\hline Essalud - Pillco Marca & Mercado Modelo - Huánuco \\
\hline UNHEVAL - Pillco Marca & Hosp. de Contingencia - la Esperanza \\
\hline Plaza Mayor - Huánuco & Plaza de la Salud - Amarilis \\
\hline Univ. de Huánuco - La esperanza & \\
\hline
\end{tabular}

\section{Muestra:}

La muestra real aplicada, se presenta en el siguiente resumen:

\begin{tabular}{|c|c|c|c|}
\hline $\begin{array}{l}\text { Catego } \\
\text { ría }\end{array}$ & Lugar de aplicación de encuestas & $\begin{array}{l}\text { Encuestas } \\
\text { efectivas }\end{array}$ & $\begin{array}{l}\text { Encuestas } \\
\text { efectivas }\end{array}$ \\
\hline \multirow{10}{*}{ 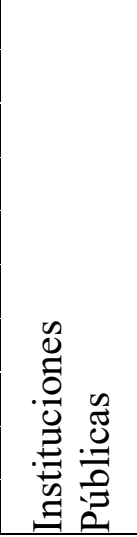 } & Seda Huánuco & 41 & \multirow{10}{*}{423} \\
\hline & Essalud & 38 & \\
\hline & Dirección Regional de Trabajo - A & 39 & \\
\hline & RENIEC & 30 & \\
\hline & Municipalidad Distrital Pillco Marca & 38 & \\
\hline & Dirección Regional de Transportes & 36 & \\
\hline & Dirección Regional de Educación & 44 & \\
\hline & Electrocentro & 42 & \\
\hline & Municipalidad Provincial Huánuco & 43 & \\
\hline & Gobernación Regional Huánuco & 36 & \\
\hline
\end{tabular}

${ }^{1}$ Se adopta esta denominación para referir la situación "inmediatamente después de hacer uso..." 


\begin{tabular}{|c|c|c|c|}
\hline & Municipalidad Distrital Amarilis & 36 & \\
\hline \multirow{3}{*}{ 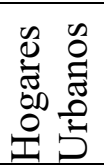 } & Distrito Huánuco & 151 & \multirow{3}{*}{334} \\
\hline & Distrito Amarilis & 110 & \\
\hline & Distrito Pillco Marca & 73 & \\
\hline \multirow{7}{*}{ 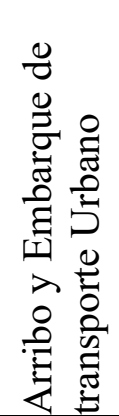 } & Essalud - Pillco Marca & 40 & \multirow{7}{*}{270} \\
\hline & UUHEVAL - Pillco Marca & 45 & \\
\hline & Plaza Mayor - Huánuco & 41 & \\
\hline & Univ. de Huánuco - La esperanza & 36 & \\
\hline & Mercado Modelo - Huánuco & 48 & \\
\hline & $\begin{array}{l}\text { Hospital de Contingencia - la } \\
\text { Esperanza }\end{array}$ & 35 & \\
\hline & Plaza de la Salud - Amarilis & 25 & \\
\hline \multicolumn{2}{|c|}{ Total muestra } & 1027 & 1027 \\
\hline
\end{tabular}

La connotación estadística de las muestras puede evaluarse a partir de la fórmula de tamaño de muestra cuando se desconoce el tamaño de la población,

$\mathrm{N}=\left(\mathrm{Z}^{2} * \mathrm{P} * \mathrm{Q}\right) / \mathrm{E}^{2}$, donde:

$\mathrm{N}=$ Tamaño de la muestra

$\mathrm{Z}=$ Valor normalizado para un nivel de confianza (NC) establecido

$\mathrm{P}=$ Probabilidad de éxito o proporción esperada

$\mathrm{Q}=$ Probabilidad de fracaso

$\mathrm{E}=$ Precisión o error máximo del resultado

Si asumimos que no existe idea de la predisposición en favor del proyecto, adoptamos $\mathrm{P}=\mathrm{Q}=0.5 ; \mathrm{NC}=95 \%(\mathrm{Z}=1.962)$. En la ecuación se conoce el tamaño de muestra trabajado, por lo que importa estimar el error esperado en las mediciones. Así, se tiene:

- Para instituciones públicas, el error de la medición es igual a 4.82\%

- Para hogares urbanos, el error de la medición es igual a 5.38\%

- Para arribos y embarques en el transporte urbano, el error de la medición es $5.97 \%$ 
Técnicas de acopio o recopilación de datos:

Procesamiento de los datos: Se utilizó la Estadística Descriptiva como apoyo, y el procesamiento electrónico de los datos recolectados se llevó a cabo utilizando plataforma Excel.

Análisis e Interpretación de los resultados. La información generada en el procesamiento de datos se analizó a partir de promedios sobre cinco (05), valoración de campo, convertido a equivalente porcentual, para expresar nivel de servicio, presentados convenientemente en cuadros resúmenes, frente al contenido del marco teórico que esta investigación ha consignado.

\section{Resultados}

Los resultados se han agrupado en tres bloques: Nivel de servicio para instituciones públicas; nivel de servicio para servicios domiciliarios y nivel de servicio para servicios extra domiciliarios. En el primer bloque, nivel de servicio institucional según dimensiones SERVQUAL, los primeros tres lugares con mejor nivel son la Dirección Regional de Transportes con 80.2\%, Dirección Regional de Trabajo con 77.7\% y RENIEC con 69.7\%; en el extremo están Gobernación Regional Huánuco con 56.3\%, Oficina de Administración de la Red Asistencial Huánuco - Essalud con 53.8\% y la Dirección Regional de Educación con 51.3\%. Por dimensiones SERVQUAL, la siguiente matriz ilustra los resultados, cada número de las celdas indica la posición institucional en la dimensión de la columna, notándose la tendencia de los tres primeros y la de los dos últimos puestos.

\begin{tabular}{|l|c|c|c|c|c|}
\cline { 2 - 6 } \multicolumn{1}{c|}{} & $\begin{array}{c}\text { Elementos } \\
\text { Tangibles }\end{array}$ & Fiabilidad & $\begin{array}{c}\text { Capac. de } \\
\text { Respuesta }\end{array}$ & Seguridad & Empatía \\
\hline Dirección Regional de Transportes & 1 & 1 & 2 & 1 & 2 \\
\hline Dirección Regional de Trabajo & 2 & 2 & 1 & 2 & 1 \\
\hline RENIEC & 3 & 3 & 3 & 3 & 3 \\
\hline Municipalidad Provincial de Huánuco & 6 & 4 & 4 & 7 & 5 \\
\hline Municipalidad Distrital de Amarilis & 7 & 5 & 5 & 6 & 4 \\
\hline Seda Huánuco & 5 & 7 & 7 & 4 & 7 \\
\hline Municipalidad Distrital de Pillco Marca & 8 & 6 & 6 & 10 & 6 \\
\hline Electrocentro & 9 & 8 & 9 & 5 & 9 \\
\hline Gobernación Regional de Huánuco & 10 & 9 & 8 & 11 & 8 \\
\hline Essalud & 4 & 11 & 11 & 8 & 10 \\
\hline Dirección Regional de Educación & 11 & 10 & 10 & 9 & 11 \\
\hline
\end{tabular}


Acerca de servicios evaluados con constructos de precios pagados e interrupciones acaecidas durante su provisión domiciliaria, energía eléctrica obtiene $44.9 \%$ de nivel de servicio y agua potable $51.2 \%$, una muy baja percepción. Los servicios extra domiciliarios evaluados en opinión domiciliaria (serenazgo y recojo de basura) y en el mismo lugar de su provisión (paraderos de transporte urbano), dieron los siguientes resultados: Serenazgo, 38.5\% de nivel de servicio, recojo de basura, $60.2 \%$ y transporte urbano, $48.0 \%$

\section{Discusión}

Se han vuelto recurrente las expresiones calidad, nivel de servicio, eficiencia y otros términos relacionados en relación a la oferta - demanda de servicios en general. Sin embargo a pesar de la masificación en su uso, resulta muchas veces difícil conceptualizarlas o simplemente definirlas, y si la hacen, éstas son muy sesgadas de su real significado; se juzga o valora solamente el resultado final o grado de satisfacción de la experiencia, sin conocer o entender los constructos que explican los juicios o valoraciones $^{2}$

Sobre las dimensiones estudiadas que enmarcadas en el modelo SERVQUAL, Alcaide (2015), de manera concreta, las define con denominaciones muy similares a las que se ha utilizado en esta investigación ${ }^{3}$ :

Tangibilidad (elementos tangibles). Apariencia de instalaciones físicas, equipo, personal, ayuda impresa y dispositivos de comunicaciones

Fiabilidad. La habilidad para desempeñar el servicio prometido de manera precisa y fiable; relacionado a la capacitación del personal para proveerlo.

Sensibilidad - respuesta (capacidad de respuesta). La buena disposición y apoyo al cliente, dotándole de un servicio oportuno; qué tan pronto comienza la verdadera relación cliente - proveedor

Garantía (seguridad). El conocimiento y cortesía de los empleados y su habilidad para expresar confianza; la probabilidad de que el resultado final en la provisión del servicio, esté exento de errores

\footnotetext{
${ }^{2}$ Quizás para el usuario no sea imperativo conocer estos aspectos

${ }^{3}$ Tomado de Evaluación del nivel de servicio en la UNHEVAL - 2017; por el autor de esta investigación
} 
Empatía. La capacidad de sentir y comprender las emociones de otros, mediante un proceso de identificación, atención individualizada al cliente

La valoración por aspecto ${ }^{4}$ entre 01 y 05 deriva en la valoración de la dimensión, y el promedio de las dimensiones da la valoración del servicio, para luego expresarlo en escala vigesimal hasta llegar al nivel de servicio en cada centro de atención, expresado en escala centesimal o porcentual. Por ejemplo en la dimensión elemento tangible para Seda Huánuco, observamos que el menor desempeño por aspecto corresponde al $4^{\circ}$ (material impreso en oficina) y el mayor desempeño al aspecto 3 (apariencia y presentación del personal); cuatro calificaciones de igual número de aspectos dan un promedio de 3.52 , y equivalencias de 14.1 en escala vigesimal y 70.3 en escala porcentual... para las dimensiones fiabilidad, capacidad de respuesta, seguridad y empatía se obtiene 57.2, 57.5, 69.5 y 62.1 respectivamente, que al promediarlos, dan un $63.3 \%$ de nivel de servicio (última columna del mencionado cuadro). Este procedimiento se repite para las demás instituciones.

Las expresiones servicio y calidad, lo mismo que nivel de servicio y calidad de servicio, pueden en ocasiones utilizarse indistintamente, sin embargo, existe la necesidad de identificar algún elemento particular en sus propias definiciones. El autor de la investigación ${ }^{5}$ define al servicio como el resultado de la interacción de bienes físicos con la actitud y aptitud de las personas que lo proveen. En esta definición, la palabra clave es resultado, como sinónimo de percepción, sensación, satisfacción. También, de las múltiples definiciones, destacamos que en general la calidad es el apego a las especificaciones en la obtención de los productos, la calidad la define el cliente, es una definición más motivadora. Ante esto, el nivel o calidad de servicio tiene necesariamente que representar o sugerir el resultado de la medición en la provisión del servicio. ¿Cuánto y sobre qué escala vale o valoramos el servicio solicitado?

\section{Nivel de servicio institucional}

De once instituciones en las cuales se ha valorado el nivel de servicio, bajo los criterios de percepción y estado general del servicio, las dos primeras ubicaciones corresponden a la Dirección Regional de Transportes con $80.2 \%$ de nivel de servicio y a la Dirección Regional de Trabajo con 77.7\%; por dimensiones, para la Dirección

\footnotetext{
${ }^{4}$ De libre elección o denominación, según el estudio en particular

${ }^{5}$ De los apuntes del curso Planeamiento y Control de Operaciones, FIIS - UNHEVAL.
} 
Regional de Transportes no pasa desapercibido el mejor resultado en las dimensiones fiabilidad y seguridad, mientras que para la Dirección regional de Trabajo este mejor desempeño corresponde a las dimensiones de empatía, elementos tangibles y seguridad $^{6}$. Acorde a la escala establecida, estos mejores niveles de servicio se juzgan como Muy Bueno (percepción) y Aceptable (estado general)

Por su parte, las dos instituciones que brindan los peores niveles de servicio son la Oficina de Administración de la Red Asistencial Huánuco - Essalud y la Dirección Regional de Educación, con 53.8\% y 51.3\% de nivel de servicio, respectivamente. Dentro de esta muy baja percepción y estado general del nivel de servicio en estas dos instituciones, en la Oficina de Administración de la Red Asistencial Huánuco Essalud, la dimensión fiabilidad tiene la más baja calificación, 38.4\%, mientras que en la Dirección Regional de Educación, esta dimensión es capacidad de respuesta, con $45.4 \%{ }^{7}$. Acorde a la escala establecida, estos peores niveles de servicio se juzgan como Regular (percepción) y Crítico (estado general). Según la complementariedad de los conceptos Nivel de Servicio y Brecha de Servicio, queda claro que a mejores niveles de servicio, corresponderán las más bajas brechas en la provisión del servicio, el caso contrario también es cierto.

Al considerar los resultados generales del nivel de servicio que proporcionan las instituciones de la muestra, el Nivel de Servicio Promedio es 63.8\%, que los ubica en la percepción de Bueno, equivalente a Alerta como estado general

\section{Nivel de Servicio y hogares urbanos}

Todos los hogares urbanos, en verdad las personas que forman parte de él, son beneficiarios, hacen uso, o requieren de otros servicios diferentes a los que se proveen en instituciones, por ello, para esta instancia de la investigación y con base en el formato SERVQUAL, se ha indagado acerca de los servicios de agua potable y energía eléctrica (servicios domiciliarios para la investigación), serenazgo, recojo de basura y transporte urbano (servicios extra domiciliarios para la investigación). Para los primeros se ha evaluado el nivel de servicio según los aspectos de precio y cortes del suministro; para los segundos la evaluación se basó en aspectos como rondas y equipamiento para Serenazgo y frecuencia y horario para el recojo de basura, apariencia

\footnotetext{
${ }^{6}$ En ambos casos considerar que los resultados por dimensión son mayores al nivel de servicio dado

${ }^{7}$ En ambos casos considerar que los resultados por dimensión son menores al nivel de servicio dado
} 
y actitud del conductor, apariencia y comodidad de la unidad de transporte, para el servicio de transporte urbano.

Los resultados para los servicios domiciliarios son decepcionantes: $44.9 \%$ de nivel de servicio para la provisión de energía eléctrica y $51.2 \%$ para la provisión de agua potable. En este caso donde los aspectos o constructos evaluados insinúan el sentido de la respuesta y la alta interacción entre personas ${ }^{8}$ es remota, a estos resultados los asumimos como tal, como una percepción, a pesar de su irrenunciable presencia y comprobada necesidad en cada hogar ${ }^{9}$. Estos resultados puestos en la escala del semáforo, los ubican en las categorías de Regular y Estado Crítico, sugiriendo inmediatas acciones orientadas a revertir la situación actual.

No son tan diferentes los resultados para los servicios extra domiciliarios: a excepción del servicio de recojo de basura que obtiene $60.2 \%$, serenazgo y transporte urbano obtienen $38.5 \%$ y $48.0 \%$ respectivamente. La valoración para serenazgo podría estar relacionada con los índices de criminalidad e inseguridad ciudadana, muy venida a menos en zonas urbanas del país; mientras que para lo obtenido en transporte urbano, parte de la explicación podría estar en relación al caos vehicular urbano y al crecimiento del parque automotor, donde la oferta de calles y áreas transitables es escasa en relación a la demanda por los mismos, sumado a ello la actitud deplorable de los propios choferes de unidades vehiculares y el estado real de sus unidades. Puestos en escala, $38.5 \%$ de valoración corresponde a una percepción de Malo y estado general del servicio Crítico - 48.0\% a una percepción de Regular y estado general Crítico

En diciembre de 2017, en un estudio de opinión pública administrado por la empresa local IP MERCADO, disponible en www.ip-mercado.com, ante el requerimiento de que el encuestado valore algunos servicios urbanos, los promedios en escala vigesimal dieron: servicio de agua potable, 13.0, que en términos porcentuales equivale a $67.0 \%$; energía eléctrica, 14.2 ó $71.0 \%$; recojo de basura, 12.3 ó 61.5\%; serenazgo, 8.4 ó 42.0\%. En el referido estudio la pregunta fue contundente, una sola valoración, mientras que en el presente se ha ponderado lo obtenido en atención en oficina y la valoración hecha en los mismos hogares.

\footnotetext{
${ }^{8}$ Característica fundamental en los servicios

${ }^{9}$ Independiente de la evaluación hecha en oficinas, donde la interrelación cliente proveedor es absoluta
} 


\section{Nivel de servicio del Transporte Urbano}

Como una extensión de la evaluación de este servicio, hecha en hogares, la investigación ha evaluado también a este servicio con base en la calificación de usuarios inmediatamente antes de usar o inmediatamente después de usar este servicio. Para este propósito se identificó puntos en la ciudad o paraderos de unidades de transporte urbano, habiéndose corroborado aquello de la baja calificación obtenido en hogares; promediando ambos resultados se obtiene que el nivel de servicio del Transporte Urbano en la ciudad de Huánuco, alcanza apenas un 46.3\% (percepción Regular y estado general Crítico). Grande es el aporte en esta baja valoración lo concerniente al conductor, explicado por su apariencia o presentación inadecuada, lo mismo que su actitud, que reflejaría una actitud indeseada. Del mismo estudio referido en el acápite anterior $^{10}$, la valoración equivalente para el transporte público urbano alcanzó 12.7 ó $63.5 \%$ en términos de nivel de servicio; las diferencias pueden explicarse por la metodología obtenida en ambas investigaciones

\section{Conclusiones}

1. De las once instituciones evaluadas, las dos mejores con mejor nivel de servicio son Dirección Regional de Transportes (80.2\%) y Dirección Regional de Trabajo (77.7\%). Las dos últimas son Oficina de Administración de la Red Asistencial Huánuco - Essalud (53.8\%) y Dirección Regional de Educación (51.3\%).

Infiriendo los resultados, el nivel de servicio promedio institucional en la ciudad de Huánuco es $63.8 \%$, con una desviación estándar de 9.3\%.

Por dimensiones, las dos mejores instituciones tienen la siguiente valoración:

Elementos tangibles: Dirección Regional de Transportes, 79.2\%; Dirección Regional de Trabajo, $78.8 \%$

Fiabilidad: Dirección Regional de Transportes, 84.2\%; Dirección Regional de Trabajo, $75.6 \%$

Capacidad de respuesta: Dirección Regional de Trabajo, 75.7\%; Dirección Regional de Transportes, $75.4 \%$

${ }^{10} \underline{w w w . i p-m e r c a d o . c o m}$ 
Seguridad: Dirección Regional de Transportes, 84.\%; Dirección Regional de Trabajo, $78.8 \%$

Empatía: Dirección Regional de Trabajo, 79.3\%; Dirección Regional de Transportes, $78.0 \%$

2. Los servicios domiciliarios Agua Potable y Energía Eléctrica, sin perjuicio de la evaluación en oficinas, obtienen niveles de servicio de $51.2 \%$ y $44.9 \%$, valores que los ubican en una percepción de Regular y en un estado general Crítico

3. Los servicios extra domiciliarios de Recojo de Basura, Transporte Urbano (ponderado de dos instancias) y Serenazgo han sido calificados con niveles de servicio de $60.2 \%, 46.3 \%$ y $38.5 \%$ respectivamente. En la escala de valoración de resultados (semáforo), el Recojo de Basura es Bueno pero en el límite inferior, el Transporte Urbano es Regular, mientras que el Serenazgo califica como malo; adicionalmente y según la misma escala, el Transporte Urbano y Serenazgo califican como en estado crítico.

\section{REFERENCIAS BIBLIOGRÁFICAS.}

Vargas, M. Q. y Aldana, L. V. Calidad y servicio, conceptos y herramientas. Bogotá Colombia: Ecoe Ediciones. 2012

Alcaide, J. C. Los 100 errores de la experiencia de cliente. España - Madrid: Editorial ESIC. 2015

Israel Gálvez, G. Calidad en la gestión de servicios. Maracaibo - Venezuela. Fondo Editorial Biblioteca. 2011

Dentón, D. Keith. Calidad en el servicio a los clientes. Madrid - España. Ediciones Díaz de Santos. 1991 